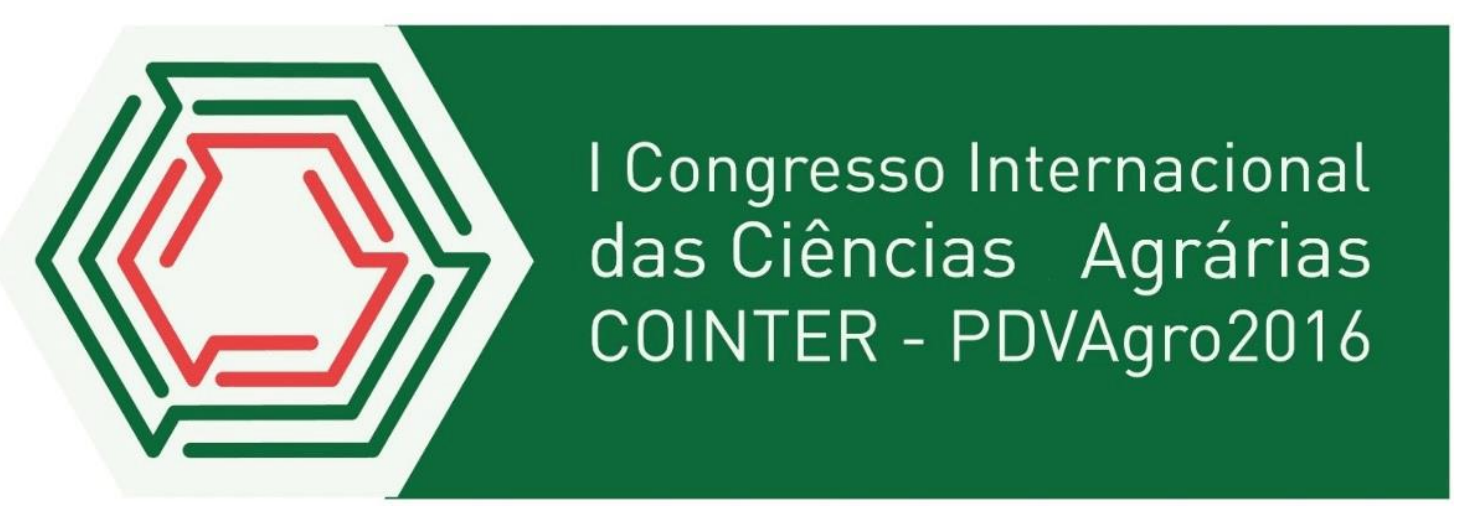

\title{
TEORIA INSTITUCIONAL E O BREVE PERCURSO DE \\ INTERNACIONALIZAÇÃO DO INSTITUTO FEDERAL DE PERNAMBUCO: PROGRAMA DESPERTANDO VOCAÇÕES E SUAS CONTRIBUIÇÕES.
}

\author{
Apresentação: Comunicação Oral
}

Maria José Cavalcante Silva; Carolayne Silva de $\mathrm{Souza}^{2}$;Edísio Raimundo Silva; Kilma da Silva Lima Viana ${ }^{4}$; Erick Viana da Silva ${ }^{5}$

\section{Resumo}

Esta pesquisa teve como objetivo mapear o percurso de internacionalização, no Instituto Federal de Pernambuco (IFPE), após a transição de Escola Agrotécnica Federal (EAF) para IFPE no município de Vitória de Santo Antão. A abordagem foi feita através de pesquisa documental e bibliográfica. A revisão de literatura é feita através do estudo da Teoria Institucional estudada por Meyer; Rowan. 1977 e sua tendência ao isomorfismo estudados por Di Maggio e Powell. Salientamos como principais resultados o Programa Despertando Vocações e a sistematização de um arcabouço de dados e informações disponíveis na instituição e que podem ser utilizados, posteriormente, como um instrumento de gestão do conhecimento institucional.

Palavras-Chave: Institutos Federais, Internacionalização, Isomorfismo, Transição.

\section{Introdução}

As mudanças organizacionais normalmente são recebidas com algumas desconfianças, já que trazem consigo alto nível de incerteza. A gestão pode observar estes elementos e de alguma forma tentar modificar tal situação, estabelecendo métodos que permitam essa conversão.

O método de adaptação depende de cada organização, por isso uma visão institucionalista sobre organizações é essencial. As organizações passaram a serem observadas de modo diferente após os primeiros trabalhos de Frederick Winslow Taylor e Henri Fayol. Taylor se preocupava em aumentar a eficiência da indústria por meio da racionalização do trabalho operário, já Fayol desenvolveu a chamada Teoria Clássica, 
que se preocupava em aumentar a eficiência da empresa por meio de suas organizações e dos princípios gerais da Administração. Mesmo tendo pontos de vista diferentes, eles estudavam maneiras de aumento de produção e, deram início aos estudos organizacionais. (OLIVER,2011; IBGP,2011)

A identificação das origens da Teoria Institucional foram identificadas no final do século XIX através das contribuições da Sociologia, que se aprofundou nos estudos pelos quais a teoria é entendida atualmente. Após a década de 40, Robert Merton e seus seguidores, esboçaram dentro da Sociologia americana, um foco maior para o que hoje se conhece por teoria institucional. A teoria institucional, coloca na essência as discussões de legitimidade da organização em tendência ao isomorfismo. $\mathrm{O}$ sucesso organizacional vai além de fatores associados à produção, eficiência e custos. Uma das estratégias identificada é a tendência de estruturas isomórficas para as organizações, ao longo do tempo alcançarem sua legitimidade e sustentação.

Segundo Di maggio e Powell (2005) podem encontrar-se no processo de isomorfismo três parâmetros: 1) isomorfismo coercitivo, que deriva de influências políticas e do problema da legitimidade; 2) isomorfismo mimético, que resulta de respostas padronizadas à incerteza; e 3) isomorfismo normativo, associado à profissionalização. Essa é uma tipologia analítica: os tipos não são sempre empiricamente distintos.

\section{Metodologia}

\section{Campo e sujeitos da pesquisa}

O campo de Pesquisa foi constituído por um campus do Instituto Federal de Pernambuco que se encontrava na condição de Escola Agrotécnica quando da criação dos Institutos Federais e como objeto de pesquisa o IFPE.

\section{Procedimentos}

Inicialmente foram analisados documentos oficiais do IFPE e revisão bibliográfica dos principais teóricos que fundamentaram a análise sob a perspectiva do isomorfismo institucional, tendo como foco a internacionalização do ensino, da pesquisa e da extensão.

\section{Resultados e Discussão}

No decorrer do desenvolvimento e transição de EAF para IF, o campus em 
análise, como toda nova estrutura organizacional decorrente da junção de EAF's e Centro Federais de Educação Tecnológica - CEFET's, passou por muitas mudanças. Logo após a assinatura da Lei 11892 de 29 de Dezembro de 2008 (BRASIL,2016), que exigia a entrega de um Plano de Desenvolvimento Institucional (IFPE,2009), onde estão contidas as metas e necessidades dos IF's projetadas ao longo de um período de tempo com o objetivo de nortear a execução das políticas institucionais foi elaborado o primeiro PDI do Instituto Federal de Pernambuco que pretendeu atuar em demandas específicas, com base nos diagnósticos apontados nessa primeira proposta de novo arranjo institucional, através de um planejamento pedagógico que se integrasse às demandas socioeconômicas e culturais da região, contribuindo de maneira determinante para que as desigualdades sociais fossem enfrentadas de maneira eficiente e construtiva. (IFPE, 2016)

O IFPE surge com uma sólida experiência de ensino na formação profissional técnica e de nível superior, com um potencial bastante promissor no âmbito da pesquisa científica e aplicada, aliando-se a isso uma enorme capacidade de desenvolvimento de ações de extensão. (IFPE,2009).

Segundo a missão do IFPE no PDI, buscou-se qualificação para os servidores apenas no âmbito nacional, o que sugere, ainda, pouca perspectiva em torno da dimensão da internacionalização, "Ser uma Instituição de referência nacional em formação profissional que promove educação, ciência e tecnologia de forma sustentável e sempre em benefício da sociedade.” (IFPE,2009).

No decorrer da análise do PDI 2009-2013, observou-se a presença de citações pontuais e implícitas acerca da internacionalização tais quais: "Promover ações de intercâmbio, parcerias, convênios e captação de recursos com instituições e empresas na área de fomento à pesquisa, ciência e tecnologia e da inovação tecnológica”. Após concluir-se o primeiro PDI, em 2014, foi criado o segundo para o período de 20142018, onde o processo de internacionalização foi evidenciado de uma forma mais direta. (IFPE, 2014)

Um tópico foi dedicado as relações internacionais, algo que até então não havia ocorrido. Em conjunto com a Arinter foram criados também a CAI (Coordenação de Acordos Internacionais, no IFPE), IsF (Inglês sem Fronteiras) e CsF (Ciência sem Fronteiras), nacionalmente, que contribuíram muito com desenvolvimento internacional da instituição. 
O novo PDI, visa quebrar as fronteiras, traz em si diversos projetos e metas a serem alcançadas em relação à internacionalização. O novo PDI trouxe a divisão de diversos conselhos, dividindo assim responsabilidades. Criou-se também um tópico dedicado ás relações internacionais. Criação do FORINTER (Fórum de Relações Internacionais das Instituições da Rede Federal de Educação Profissional, Científica e Tecnológica) e o CONIF - Conselho Nacional das Instituições da Rede Federal de Educação Profissional, Científica e Tecnológica que ajudados pela ARINTER puderam expandir as relações internacionais. (IFPE 2014).

Uma das metas também requer o desenvolvimento das CELEs - Centro de Língua Estrangeira do Instituto Federal de Pernambuco, com cursos de língua estrangeira, o que significa mais qualificação para os estudantes. (IFPE,2012)

Criação de algumas coordenações com âmbito internacional, como o CAI - Coordenação de Acordos Internacionais, IsF -Coordenação do Idioma sem Fronteiras.

Reconhecimento da Assessoria de Relações Internacionais - ARINTER e suas atribuições, como desenvolver as ações necessárias à eficiente representação do IFPE em congressos, reuniões, simpósios, seminários, cursos e eventos de caráter internacional, bem como providenciar a divulgação dos resultados decorrentes desses eventos. (IFPE, 2012;2014).

Diversas metas no âmbito internacional, Mobilidade de estudantes e servidores; Acordos interinstitucionais, entre outros. É de extrema importância a criação dos PDIs, porque organizam as ações, de modo que sejam executadas a partir de um cronograma e planejamento previamente estabelecidos.

Anteriormente à criação dos IF's houve o estabelecimento, por portaria, de um assessor de relações internacionais no âmbito do CEFET. No entanto, um passo importante foi dado em 2012 com a criação da ARINTER,

A partir de 2012 foi criada e estruturada a Assessoria de Relações Internacionais, com os seguintes objetivos:

$\begin{array}{ll}\text { Proporcionar } & \text { visibilidade às ações dos Institutos Federais, em âmbito } \\ \text { nacional e } & \text { internacional; Promover atividades que proporcionem a } \\ \text { ampliação } & \text { dos processos de cooperação e de intercâmbio internacionais } \\ \text { dos Institutos } & \text { Federais, nas áreas de ensino, pesquisa e extensão; Estimular } \\ \text { a inserção } & \text { internacional dos Institutos Federais, visando ao } \\ \text { aperfeiçoamento e à qualificação dos estudantes, professores e técnicos } \\ \text { administrativos destas instituições e das instituições parceiras } \\ \text { internacionais (IFPE, 2013.1). }\end{array}$


Uma outra instância importante surgida juntamente com os Institutos Federais foi o Fórum de Relações Internacionais - FORINTER (FORINTER,2009), que foi criado em uma reunião, convocada pela Secretaria de Educação profissional e Tecnológica - SETEC, realizada em Brasília em 2009. Além da criação do FORINTER, estava em pauta, a elaboração da Política de Relações Internacionais e do plano de Trabalho 2009/2010 do mesmo.

Ao verificarmos os documentos institucionais da Assessoria de Relações Internacionais obteve-se informações que através de uma cronologia de fatos permitiram o melhor entendimento das consequências nos âmbitos nacionais e internacionais, da aplicação dessas iniciativas de estruturação sistêmica de uma política de relações internacionais. Uma ação de relevância foram as missões internacionais realizadas em âmbito nacional nos IF's assessoradas pelo FORINTER. No caso em particular e em especial no IFPE, no primeiro momento com a missão Argentina, Chile e Portugal. O Brasil tem um papel importante no relacionamento com as instituições da Comunidade dos Países de Língua Portuguesa - CPLP, por ser responsável por muitas das iniciativas.

\footnotetext{
Em consonância com essas orientações, o IFPE iniciou em 2012 aproximação com Instituições de Ensino Técnico e Superior, com vistas a estabelecer cooperação internacional. Argentina, Chile e Portugal inicialmente foram visitados e foram assinados acordos iniciais de cooperação. Como consequência dos acordos firmados, em 2013.1 missões com representantes de todos os campi do IFPE e de áreas específicas do conhecimento foram realizadas, com objetivo de iniciar a cooperação e ações práticas.

Foram firmados acordos de cooperação com as seguintes instituições: Universidad Nacional de Cuyo, Universidad de Mendoza, Universidad NAcional de La Plata, Instituto Tecnologico Universitario, INACAP, Universidad de Playa Ancha, Universidad Tecnologica Metropolitana, Universidad Nacional Aberta y a Distancia Colômbia, Institutos Politécnicos de Setubal, Santarém, Bragança, Guarda, Castelo Branco e Porto. (IFPE,2013.1)
}

No segundo momento, a Missão Panamá, por representar um passo de relação de cooperação na qual ocorreu a transferência de experiência dos IF's na criação dos Institutos Tecnológicos Superiores do Panamá - ITS.

O Instituto Federal de Educação Ciência e Tecnologia de Pernambuco, recebeu através da Secretaria do Governo do Estado de Pernambuco em Julho de 2013 a visita da missão da delegação panamenha composta pelo Diretor de Educação Técnica Profissional do Ministério da Educação do Panamá e responsável pela criação dos Institutos Tecnológicos Superiores - ITS, e do Vice-reitor da Universidade Tecnológica 
do Panamá - UTP, juntamente com a Gerente Geral para Setor Privado e Terceiro Setor de Pernambuco. (IFPE, 2013.2)

No ano de 2013, através de uma parceria com a Universidade Nacional de La Plata foram recebidos 06 estudantes em mobilidade internacional em diversos campi do IFPE. (IFPE, 2013b) Os estudantes possuíam vinculação com o campus em estudo e cursavam disciplinas além de ministrarem cursos livres de idiomas.

Em particular, na transição de EAF para IF, no campus em estudo, pode-se observar que decorrente de diversos motivos, dentre eles a expansão dos Campi, com uma nova identidade, e um movimente crescente de perda de contato com as comunidades rurais e trabalhos de extensão pontuais, foram criados alguns mitos nas comunidades vizinhas, como por exemplo, de que a instituição era particular e que apenas pessoas com maior poder aquisitivo poderiam ter acesso a ela. Outro mito era em relação aos cursos, que seriam voltados para o trabalho braçal. Dessa forma, paulatinamente o perfil dos estudantes mudou de agrário para urbano. (SOUZA,2009) Entre 2014 e 2015 no campus em pauta foram criados dois programas internacionais com objetivo de despertar o interesse dos estudantes por carreiras ligadas às ciências agrárias e licenciaturas. Assim sendo, ações locais, nacionais e internacionais começaram, dentro do IFPE, a serem fomentadas pela iniciativa da comunidade de servidores e estudantes com objetivo de despertar o interesse por essas carreiras profissionais, criar e fortalecer vínculos interinstitucionais e internacionais.

\section{Considerações}

O trabalho apresenta os objetivos propostos com um relato da trajetória do processo de internacionalização no IFPE e se mostra relevante pela necessidade de maior conhecimento sobre os processos históricos de formação da realidade institucional. Observamos que houve mudanças significativas através dos documentos relatados. Os CELE's as Missões Panamá, Argentina, Chile e Portugal, por exemplo, merecem destaque como atividades que muito contribuíram para o processo de internacionalização no IFPE e, que esse processo foi uma estratégia de diminuir 'fronteiras' no que se diz respeito, inclusive, a menção do processo de internacionalização nos documentos oficiais. Iniciativas decorrente desse processo inicial de trabalho sistêmico iniciado através da SETEC/MEC, FORINTER e 
ARINTER/IFPE geraram condições mínimas para que programas de dimensões internacionais pudessem germinar no campus agrícola estudado. Sugere-se que, em trabalhos posteriores possa ser investigado e comparado o comportamento de outros campi do IFPE e também em outros Institutos Federais para identificação e maior compreensão de possíveis variáveis que interfiram na consolidação de processos de internacionalização. De início observamos um certo receio das organizações e aceitar o modelo e, somente através de ações como visitas internacionais e a iniciação de políticas de internacionalização houve uma maior aceitação, além disso, de início a mudança estava voltada ao âmbito nacional e só depois com o decorrer do tempo o âmbito internacional foi sendo mais visado, mais explorado.

\section{Referências}

BRASIL. Lei 11.892 de 29 de Dezembro de 2008. Disponível em http://www.planalto.gov.br/ccivil_03/_ato2007-2010/2008/lei/111892.htm. Acesso em: 08 out. 2016.

DI MAGGIO \& POWELL.A gaiola de ferro revisitada: isomorfismo institucional e racionalidade coletiva nos campos organizacionais. [S.1.]: RAE-Revista de Administração $\quad$ de $\quad$ Empresas, $2005 . \quad$ Disponível em:<http://www.fgv.br/rae/artigos/revista-rae-vol-45-num-2-ano-2005-nid44775/>. Acesso em:08 out. 2016.

FORINTER. Fórum de Relações Internacionais dos Institutos Federais de Educação, Ciência e Tecnologia: proposta de política de internacionalização Brasília, 2009. 4 p.

IBGP - INSTITUTO BRASILEIRO DE GOVERNANÇA PÚBLICA. Geraldo Loureiro. A HISTORIA DA ADMINISTRAÇÃO: DE TAYLOR E FAYOL ATÉ A PÓS MODERNIDADE. Disponível em: <http://academiamarketing.blogspot.com.br/2011/11/historia-da-administracao-detaylor-e.html>. Acesso em: 05 ago. 2016.

IFPE - INSTITUTO FEDERAL DE EDUCAÇÃO, CIÊNCIA E TECNOLOGIA DE PERNAMBUCO. Centro de Línguas Estrangeiras do Instituto Federal de Educação, Ciência e Tecnologia de Pernambuco Resolução CONSUP/IFPE Nº 04 de 2013, Recife,PE, 2013. 13 p. 
IFPE - INSTITUTO FEDERAL DE EDUCAÇÃO, CIÊNCIA E TECNOLOGIA DE PERNAMBUCO. Reitoria. Histórico do ifpe. Disponível em: <http://www.ifpe.edu.br/o-ifpe/reitoria/historico>. Acesso em: 05 ago. 2016.

IFPE - INSTITUTO FEDERAL DE EDUCAÇÃO, CIÊNCIA E TECNOLOGIA DE PERNAMBUCO. Plano de Trabalho Assessoria de Relações Internacionais. Política de Relações Internacionais dos Institutos Federais de Educação, Ciência e Tecnologia. Reitoria Instituto Federal de Pernambuco, Recife, 2012. 11 p.

IFPE - INSTITUTO FEDERAL DE EDUCAÇÃO, CIÊNCIA E TECNOLOGIA DE PERNAMBUCO. Projeto Político Pedagógico. Recife,2012a. 95 p.

IFPE - INSTITUTO FEDERAL DE EDUCAÇÃO, CIÊNCIA E TECNOLOGIA DE PERNAMBUCO. Relatório das Missões Internacionais: Argentina, Chile e Portugal 2013.1. Recife, 2013.1

IFPE - INSTITUTO FEDERAL DE EDUCAÇÃO, CIÊNCIA E TECNOLOGIA DE PERNAMBUCO.Relatório das Missões Internacionais: Panamá 2013.2. Recife, 2013. 2

IFPE - INSTITUTO FEDERAL DE EDUCAÇÃO, CIÊNCIA E TECNOLOGIA DE PERNAMBUCO. Relatório Sintético das Ações da Arinter. Recife, 2013a.

IFPE - INSTITUTO FEDERAL DE EDUCAÇÃO, CIÊNCIA E TECNOLOGIA DE PERNAMBUCO. Programa de Acolhimento ao Visitante Internacional. Resolução CONSUP/IFPE No 05 de 2013, Recife,PE, 2013b. p.4.

IFPE - INSTITUTO FEDERAL DE EDUCAÇÃO, CIÊNCIA E TECNOLOGIA DE PERNAMBUCO.Ministério da Educação. Plano de Desenvolvimento Institucional. 1. ed. Recife: [s.n.],[2009-2013]. 366 p.

IFPE - INSTITUTO FEDERAL DE EDUCAÇÃO, CIÊNCIA E TECNOLOGIA DE PERNAMBUCO.Ministério da Educação. Plano de Desenvolvimento Institucional. 2. ed. Recife: [s.n.],[2014-2018]. 283 p.

OLIVER, Paula. A HISTORIA DA ADMINISTRAÇÃO: DE TAYLOR E FAYOL ATÉ A PÓS MODERNIDADE: acadêmicos marketing. Disponível 
em:<http://academiamarketing.blogspot.com.br/2011/11/historia-da-administracao-detaylor-e.html>. Acesso em: 07 out.2016.

SOUZA,C.A.de. Princípios Agroecológicos na Formação do Técnico em Agropecuária: Estudo de caso da Escola Agrotécnica Federal de Vitória de Santo Antão-PE 2009. 73 f. Dissertação (Mestre em Ciências) - Universidade Federal Rural do Rio de Janeiro, Rio de Janeiro, 2009. 